\title{
Relativistic bottomonium spectrum from anisotropic lattices *
}

\author{
X. Liao and T. Manke ${ }^{\mathrm{a}}$ \\ aPhysics Department, Columbia University, New York, NY 10027, USA
}

We report on our results from a fully relativistic simulation of the quenched bottomonium spectrum. Using an anisotropic formulation of Lattice QCD, we were able to retain a very fine resolution into the temporal direction for a range of different lattice spacings. At fixed renormalized anisotropy we study the scaling properties of the spectrum and compare our results with non-relativistic calculations.

\section{INTRODUCTION}

The non-perturbative study of heavy quark systems is complicated by the large separation of momentum scales which are difficult to accommodate on conventional isotropic lattices. Several approximations to relativistic QCD have been proposed to describe accurately the low energetic phenomenology of heavy quarkonia [1]:2]. However, the (non-)perturbative control of systematic errors in those approximations is very difficult and in practise one has to rely on additional approximations. The high precision results for the spin structure in non-relativistic bottomonium calculations have been hampered by large systematic errors which are difficult to control within this effective theory. Higher order radiative and relativistic corrections are sizeable for bottomonium and even more so for charmonium. Still more cumbersome are large observed scaling violations [3] that cannot be controlled by taking the continuum limit.

We take this as our motivation to study heavy quarkonia on anisotropic lattices in a fully relativistic framework. This approach has previously been used to calculate the charmonium spectrum with unprecedented accuracy [4 [6]. Here we extent the method to even heavier quarks and focus on our new results for bottomonium. Preliminary results were reported in [6]. In Section 2 we briefly review our action and simulation parameters and Section 3 discusses our results.

\footnotetext{
*This work was conducted on the QCDSP machines at Columbia University and RIKEN-BNL Research Center. TM and XL are supported by DOE.
}

\section{ANISOTROPIC LATTICES}

To study the relativistic propagation of heavy quarks it is mandatory to have a fine resolution in the temporal lattice direction. To this end we employ an anisotropic gluon action:

$S=-\beta\left(\sum_{x, \mathrm{i}>\mathrm{j}} \xi_{0}^{-1} P_{\mathrm{ij}}(x)+\sum_{x, \mathrm{i}} \xi_{0} P_{\mathrm{it}}(x)\right)$.

This is the standard Wilson action written in terms of simple plaquettes, $P_{\mu \nu}(x)$. Here $\left(\beta, \xi_{0}\right)$ are two bare parameters, which determine the gauge coupling and the renormalized anisotropy, $\xi=a_{s} / a_{t}$, of the quenched lattice. The anisotropic gluon action (11) is designed to be accurate up to $\mathcal{O}\left(a_{s}^{2}, a_{t}^{2}\right)$.

For the heavy quark propagation in the gluon background we used the "anisotropic clover" formulation as first described in [4, 5]. The discretized form of the continuum Dirac operator, $Q=m_{q}+\not D$, reads

$$
\begin{aligned}
Q= & m_{0}+\nu_{s} W_{i} \gamma_{i}+\nu_{t} W_{0} \gamma_{0}- \\
& \frac{a_{s}}{2}\left[c_{s} \sigma_{0 k} F_{0 k}+c_{t} \sigma_{k l} F_{k l}\right], \\
W_{\mu}= & \nabla_{\mu}-\left(a_{\mu} / 2\right) \gamma_{\mu} \Delta_{\mu} .
\end{aligned}
$$

The five parameters in Eq. 2 are all related to the quark mass, $m_{q}$, and the gauge coupling as they appear in the continuum action. Their classical estimates have been given in [5]. Here we chose $m_{0}$ non-perturbatively, such that the rest energy of the hadron corresponds to its experimental value $\left(M\left({ }^{3} S_{1}^{--}\right)=9.46 \mathrm{GeV}\right)$. We also 
fix $\nu_{s}=1$ and adjust $\nu_{t}$ non-perturbatively for the mesons to obey a relativistic dispersion relation $(c(\mathbf{0})=1)$ :

$E^{2}(\mathbf{p})=E^{2}(\mathbf{0})+c^{2}(\mathbf{p}) \mathbf{p}^{2}+\mathcal{O}\left(\mathbf{p}^{4}\right) \ldots$.

For the clover coefficients $\left(c_{s}, c_{t}\right)$ we choose their classical estimates and augment this prescription by tadpole improvement. The tadpole coefficient has been determined from the average link in Landau gauge: $u_{\mu}=1 / 3\left\langle U_{\mu}(x)\right\rangle_{\text {Landau }}$. Any other choice will give the same continuum limit, but with this prescription we expect only small $\mathcal{O}(\alpha a)$ discretization errors.

Using the anisotropic gluon action (11) we generate several thousand quenched gauge field configuration for a variety of lattice spacings and anisotropies. Every few hundred sweeps we calculate relativistic quark propagators from the inverse of Eq. 2. This leaves us with several hundred independent measurements for each set of bare parameters. The construction of meson correlators with definite $J^{P C}$ from quark propagators is standard [6]. Our fundamental bilinears are constructed from two 4-spinors, one of $16 \Gamma$ matrices and additional insertions of up to 2 lattice derivatives:

$M(x)=\bar{q}(x) \Gamma_{i} \Delta_{j} \Delta_{k} q(x)$.

We have further improved those operators to give states with different projection onto the ground state. Here we follow [7] and employ a combination of various quark smearings and APEsmearing for the gauge links. In practise we find it often useful to use gauge-fixed box sources, where we can vary the overlap by simply varying the extent of the box. This allows us to extract reliably both the ground state energies and their excitations from correlated multi-state fits to several channels. We demand consistency for fit results from different fit methods and fit ranges. In order to call a fit acceptable we require its Q-value to be bigger than 0.1 . In Table 1 we list the parameters of our simulation.

\section{BOTTOMONIUM SPECTRUM}

In Figure 1 we show the continuum limit of our results and selected exotic candidates in the bottomonium spectrum.
The later are results from only one lattice spacing at $(6.1,4)$. To convert our lattice results into dimensionful quantities we used the ${ }^{1} P_{1}-{ }^{3} S_{1}$ splitting which is $\approx 440 \mathrm{MeV}$ experimentally. It is well-known that, without dynamical sea quarks in the gluon background, the definition of the lattice spacing is ambiguous and one cannot reproduce all experimental splittings simultaneously. For the purpose of this work we admit to the uncertainties of the quenched calculation and focus on other systematic errors instead.

As mentioned above we are tuning $\nu_{t}$ nonperturbatively at each mass, so as to reproduce a relativistic dispersion relation to within $1-2 \%$. We tested for systematic errors on spectral quantities as we vary $\nu_{t}$ and find that they are small, $<$ $1 \%$. We have also performed a finite volume study and find that volumes with $L \approx 1 \mathrm{fm}$ are sufficiently large to accommodate the small bottomonium ground states. For even smaller volumes we could observe some shifts in the spectrum, namely the hyperfine splitting is suppressed.

The most interesting aspect of our work is the determination of the spin-structure in bottomonium from a relativistic formulation. In Fig. 2 we show the continuum extrapolation of the hyperfine splitting along with selected results from non-relativistic calculations. Clearly there are large scaling violations which result in a rather large continuum limit with sizeable errors. We also notice some deviation from quenched nonrelativistic calculations at finite lattice spacing. We interpret those discrepancies as due to higher order relativistic corrections as well as a differ-

Table 1

Simulation Parameters. Using ${ }^{1} P_{1}-{ }^{3} S_{1}$ to set the scale, we adjust the bare quark mass such that the $1^{--}$corresponds to the experimental values in $b \bar{b}(\Upsilon)$.

\begin{tabular}{cccc}
\hline$(\beta, \xi)$ & $N_{s}^{3} \times N_{t}$ & configs & $1^{--}[\mathrm{GeV}]$ \\
\hline$(5.7,4)$ & $8^{3} \times 64$ & 291 & $10.58(67)$ \\
$(5.9,4)$ & $8^{3} \times 64$ & 600 & $9.25(61)$ \\
$(6.1,4)$ & $16^{3} \times 96$ & 660 & $10.40(30)$ \\
$(6.3,4)$ & $16^{3} \times 128$ & 450 & $12.60(67)$ \\
$(6.5,4)$ & $16^{3} \times 160$ & 710 & 13.04() \\
$(5.9,5)$ & $8^{3} \times 96$ & 780 & $9.57(44)$ \\
$(6.1,5)$ & $16^{3} \times 128$ & 370 & $10.11(97)$ \\
\hline
\end{tabular}


ent tadpole prescriptions in [8]. In Refs. [3,9] improved gluon actions were used which can also result in different scaling violations. From potential models and results in [3], we should expect dynamical calculations to raise the hyperfine splitting even further. We also like to point out the very good agreement for our simulations with different renormalized anisotropy (4 and 5). This indicates that we are working already close to the Hamiltonian limit of lattice QCD.

For the fine structure, Fig. 3, we observe a closer agreement with NRQCD calculations. However, the large scaling violations result in an overestimate of the experimental value by $3 \sigma$.

For the first excited states, $2 S$, we also find an enlargement compared to experiment and in line with previous estimates of this quantity. We feel that anisotropic lattices provide a more reliable tool to resolve such high excitation, but we leave a more detailed analysis to future work.

In conclusion, we have demonstrated that a relativistic simulation of bottom quarks is possible on anisotropic lattices. Work is in progress to treat also light dynamical quarks in an anisotropic fashion.

\section{REFERENCES}

1. B. Thacker and G.P. Lepage, Phys. Rev. D43 (1991) 196; G.P. Lepage et al., Phys. Rev. D46 (1992) 4052; C.T.H. Davies et al., Phys. Rev. D50 (1994) 6963.

2. A.X. El-Khadra et al., Phys. Rev. D55 (1997) 3933.

3. CP-PACS Collaboration, T. Manke et al., Phys. Rev. D62 (2000) 114508.

4. T.R. Klassen, Nucl. Phys. B (Proc. Suppl.) 73 (1999) 918.

5. P. Chen, Phys. Rev. D64 (2001) 034509.

6. P. Chen et al., Nucl. Phys. B (Proc. Suppl.) 94 (2001) 342.

7. CP-PACS Collaboration, T. Manke et al., Phys. Rev. Lett. 82 (1999) 4396.

8. C.T.H. Davies, et al., Phys. Rev. D58 (1998) 054505.

9. CP-PACS Collaboration, T. Manke et al., Nucl. Phys. B (Proc. Suppl.) 86 (2000) 397.

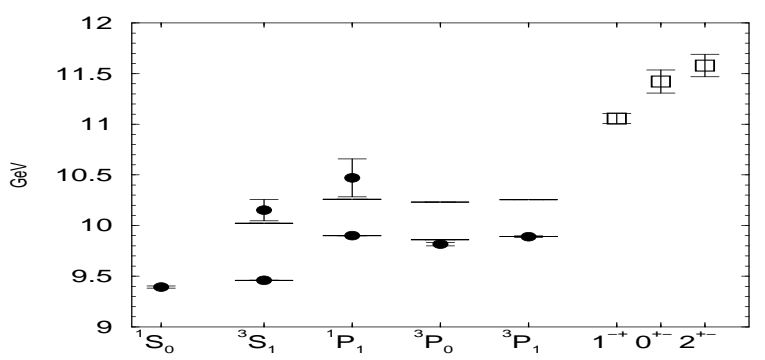

Figure 1. Quenched bottomonium spectrum. We plot continuum estimates as full circles. Selected results for exotic candidates (hybrids) from finite lattice spacing are shown as open squares. The ${ }^{1} P_{1}-{ }^{3} S_{1}$ splitting is used to set the scale.

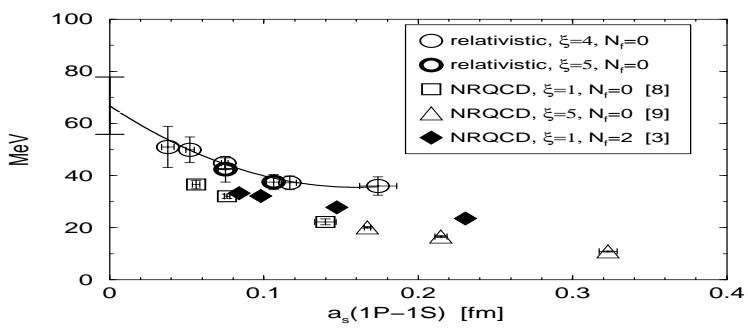

Figure 2. Hyperfine splitting. We show the continuum extrapolation from a linear-plusquadratic ansatz in $a_{s}$. 


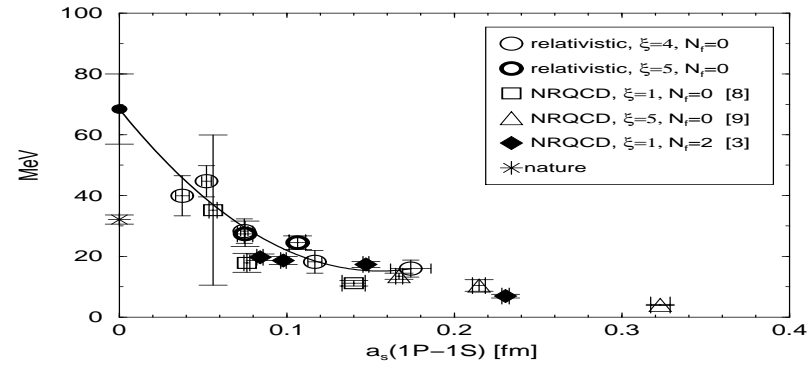

Figure 3. Fine structure. As in Fig. 2. 